\title{
Eyeball deviation by orbital mucocele after midface sinus injury
}

\author{
Se Young Oh, \\ Ji Seon Choi, \\ Jin Soo Lim, \\ Min Cheol Kim \\ Department of Plastic and \\ Reconstructive Surgery, St. Vincent's \\ Hospital, College of Medicine, The \\ Catholic University of Korea, Suwon, \\ Korea
}

A mucocele is an epithelium-lined, mucus-filled cavity in the paranasal sinuses. Mucocele may develop due to scarring and obstruction of the sinus ostium caused by midface sinus trauma, such as orbital bone fracture or endoscopic sinus surgery. The authors report two cases of orbital mucocele as complications following midface sinus injury (endoscopic sinus surgery in one case, and orbital fracture repair in the other). In both cases, imaging studies showed a large orbital mucocele accompanied by bony erosion and orbital wall remodeling, compressing the ocular muscle. Using an open approach, the lesion was excised and marsupialized. The symptoms resolved, and the postoperative eyeball position was normal. Orbital mucocele may cause serious complications such as ocular symptoms, orbital cellulitis, osteomyelitis, and the formation of an abscess with the potential to invade the brain. Therefore, surgeons should consider the possibility of mucocele as a late complication of surgery and initiate an immediate work-up and surgical treatment if needed.

Keywords: Deviation / Mucocele / Orbital / Sinus surgery

\section{INTRODUCTION}

Mucocele occurs as a result of blockage of the normal mucus pathway, which can be caused by trauma, chronic inflammation, allergies, and masses. Cysts expand when the normal drainage pathway is blocked, and fluid is secreted from the epithelium in the sinuses. The pressure from an expanding cyst causes absorption or remodeling of the surrounding bone tissue. Mucocele is known as a rare complication of frontal sinus trauma [1,2]. However, very few cases of mucocele have been reported after trauma of the midface sinus, including endoscopic sinus surgery and orbital fractures [3]. In the literature, the interval between injury and the development of clinically

Correspondence: Min Cheol Kim

Department of Plastic and Reconstructive Surgery, St. Vincent's Hospital, College of Medicine, The Catholic University of Korea, 93 Jungbu-daero, Paldal-gu, Suwon 16247, Korea

E-mail: wisethoth@hotmail.com

Received October 14, 2019 / Revised December 4, 2019 / Accepted December 6, 2019 apparent mucocele has been reported to range from 1 to 35 years $[4,5]$.

Here, the authors report two cases of orbital mucocele as complications following midface sinus injury. The first case occurred in a patient who had undergone endoscopic sinus surgery more than 10 years previously and presented with proptosis and an orbital mass. In the second case, a patient who had undergone orbital wall fracture repair using an alloplastic implant several years earlier presented with exophthalmos and ocular pain that had lasted for a few months. We performed imaging studies and completely removed the mucocele using an open approach.

\section{CASE REPORTS}

\section{Case 1}

A 28-year-old woman presented to the outpatient clinic of the department of ophthalmology with a small hard mass in the 
right medial upper eyelid that had lasted for 2 years. The patient had undergone endoscopic sinus surgery 12 years previously and had no other history of surgery or trauma. At the ophthalmology outpatient clinic, she received no definitive diagnosis and did not undergo treatment. After 2 years, the mass became larger and was accompanied by pain. For these symptoms, she visited the ophthalmology outpatient clinic again and the ophthalmologist referred her to the outpatient clinic of the department of plastic and reconstructive surgery. About 4 days prior to presentation, she had experienced swelling in her right eye area, frequent secretion of tears, and severe headaches. On physical examination, a palpable mass was observed in the right upper medial eyelid area. The mass was soft but fixed, and the patient complained of sensory disturbances in the right supraorbital nerve region. On an ophthalmic examination, the degree of exophthalmos was $21 \mathrm{~mm}$ on the right side and $17 \mathrm{~mm}$ on the left side, and eye protrusion and lateral displacement of

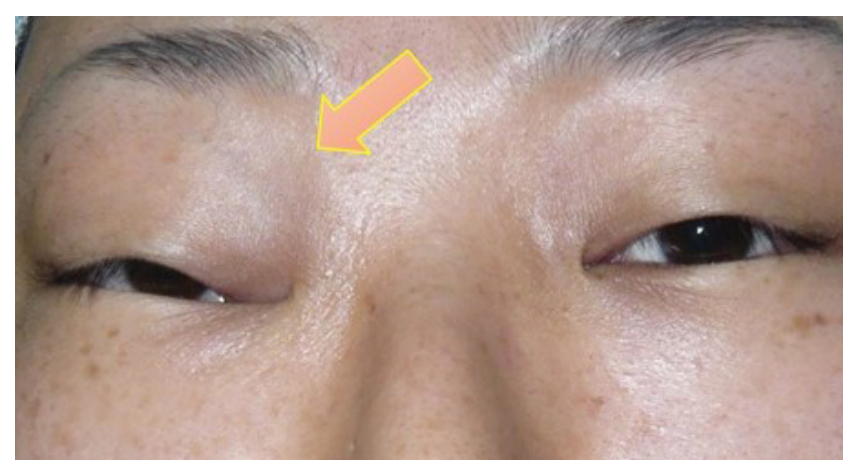

Fig. 1. Preoperative clinical photograph of case 1. A preoperative photograph shows proptosis and a protruding mass on medial canthal area of the right eye (arrow). The right eyeball showed lateral deviation.
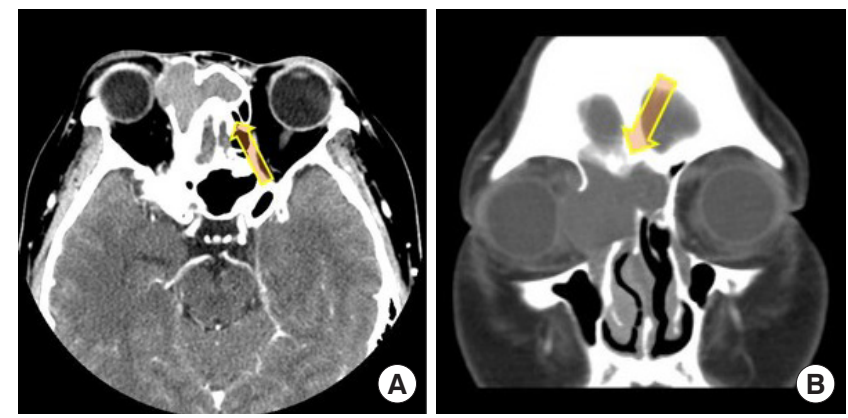

Fig. 2. Preoperative enhanced computed tomography of case 1 . The images show an expanding soft tissue lesion $(3.8 \times 2.8 \mathrm{~cm})$ in the right ethmoid sinus and bilateral frontal sinuses (arrow) that extended into the right orbit. The right eyeball was displaced laterally and bony remodeling was noted in the right medial wall of the orbit, which was pushed into the orbital contents. The cyst was suspected to be mucocele. No intracranial invasion was noted. (A) Axial plane and (B) coronal plane. the right eyeball were observed (Fig. 1). Computed tomography (CT) was performed, and an expanding cyst measuring $3.8 \times 2.8 \mathrm{~cm}$ was observed over the frontal and right ethmoid sinuses (Fig. 2). In addition, magnetic resonance imaging (MRI) was performed, and cystic fluid filling the frontal and right ethmoid sinuses was found to have invaded the right orbit. In order to perform a radical excision of the mucocele and to reconstruct the displaced orbital bone, a skin incision was made, and the mucocele was removed completely (Fig. 3). Next, we observed the medial orbital wall through the nasal cavity using an endoscope, and observed that although the protuberance had been removed, severe adhesions remained. Marsupialization was performed to drain the fluid from the remnant cyst in the ethmoid and frontal sinuses. Resection of the medial orbital wall was planned for the future in an attempt to avoid the risk of infection that would accompany immediate reconstruction. After surgery, lateral displacement of the eyeball and right-side exophthalmos resolved. At 5 days after surgery, the patient was discharged with no complications. A follow-up CT scan at

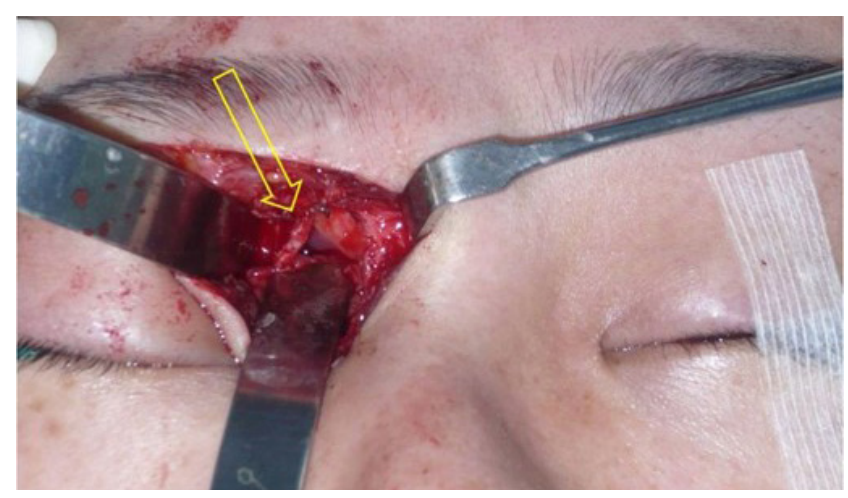

Fig. 3. Intraoperative clinical photograph of case 1. We used a Lynch incision to completely expose the orbital cyst. The mucocele was radically excised as much as possible across the orbit, ethmoid sinus, and frontal sinus. After excision of the mucocele, bony remodeling of the medial orbital wall was observed (arrow).
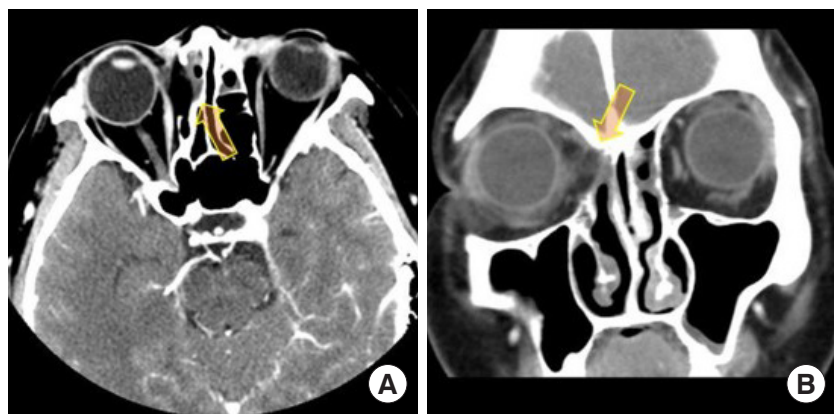

Fig. 4. Postoperative enhanced computed tomography of case 1. Follow-up computed tomography shows bony remodeling of the right medial orbital wall and removal of the mucocele (arrow). (A) Axial plane and (B) coronal plane. 
postoperative 5 months showed complete removal of the mucocele with no specific complications (Fig. 4). The patient was followed for 10 months, and no noteworthy complications or symptoms were observed.

\section{Case 2}

A 59-year-old man presented to the outpatient clinic of the department of plastic and reconstructive surgery with progressive exophthalmos. He had a history of a medial and orbital floor blow-out fracture 8 years previously and had undergone surgery to reconstruct the orbital wall using Medpor (porous polyethylene) 1 week after the trauma. A clinical examination confirmed left-side exophthalmos (Fig. 5). The patient stated that he had not experienced visual and sinus-related symptoms or nasal obstruction. CT showed a high-density, fusiform, extraconal mass encasing the Medpor on the left orbital floor (Fig. 6). The patient was scheduled for elective excision of a suspected mucocele. During surgery, a cyst was found to be strongly attached to the previously inserted Medpor, under which a thick layer appeared to have been formed by granulation tissue. Using a periosteal elevator, the Medpor, mucocele, and granula-

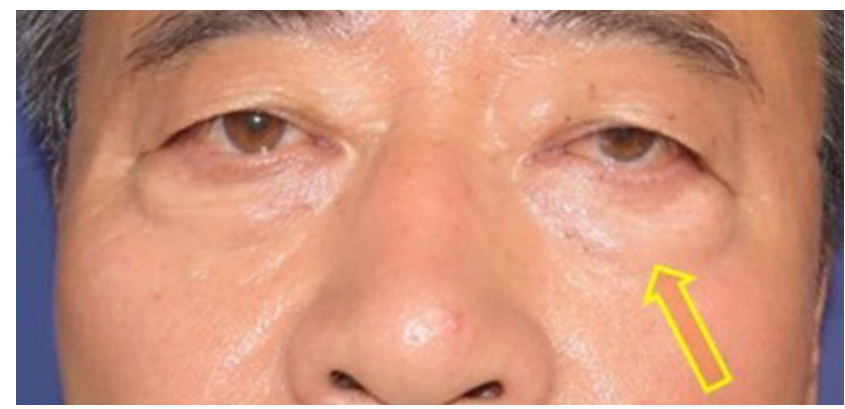

Fig. 5. Preoperative clinical photograph of case 2 shows mild exophthalmos of the left eyeball and bulging of the left lower eyelid (arrow).
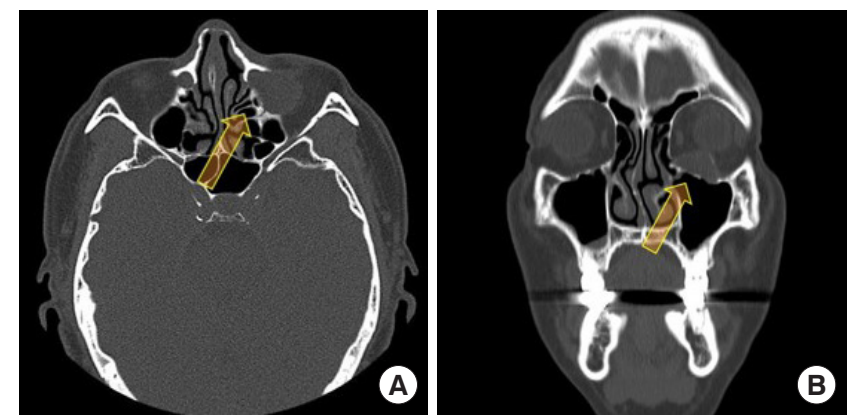

Fig. 6. Preoperative computed tomography images of case 2. Preoperative computed tomography showed an orbital cyst $(2.5 \times 1.8 \mathrm{~cm})$ (arrow). The left inferior rectus muscle was compressed by this mass. In the coronal section, the orbital floor seemed to be concealed by the healed fracture, unlike the right side, which was patent. (A) Axial plane and (B) coronal plane. tion tissue layer were separated and completely removed (Fig. 7). The lesion and discharge samples were sent for a histopathological examination and culture/sensitivity testing, respectively. No intraoperative or postoperative complications occurred. The patient's preoperative symptoms improved dramatically during the first few postoperative days, and the patient was discharged in a healthy condition on day 5 , when a CT image showed complete absence of the mucocele (Fig. 8). The patient was followed regularly for 1 year, during which no evidence of exophthalmos or limitation of eyeball movement was noted.

\section{DISCUSSION}

Orbital mucocele can occur in any paranasal sinus and cause ophthalmic symptoms. In the paranasal sinuses, mucocele most

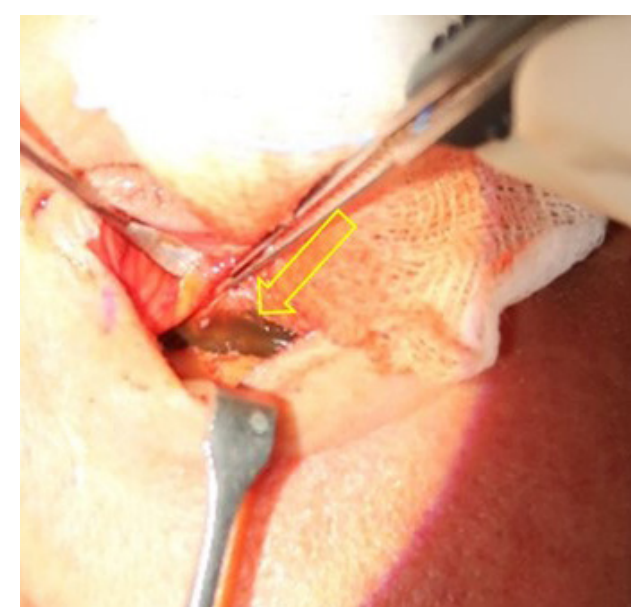

Fig. 7. Intraoperative clinical photograph of case 2. Through a subciliary approach, a brownish cyst suspected to be an orbital mucocele was exposed (arrow). A thick brownish discharge was noted pouring from the lesion and was aspirated. Next, the remnant cyst wall was completely removed. We removed the previously inserted Medpor due to the possibility of infection. The previous orbital fractures were functionally covered by a membrane consisting of dense fibrous tissue.
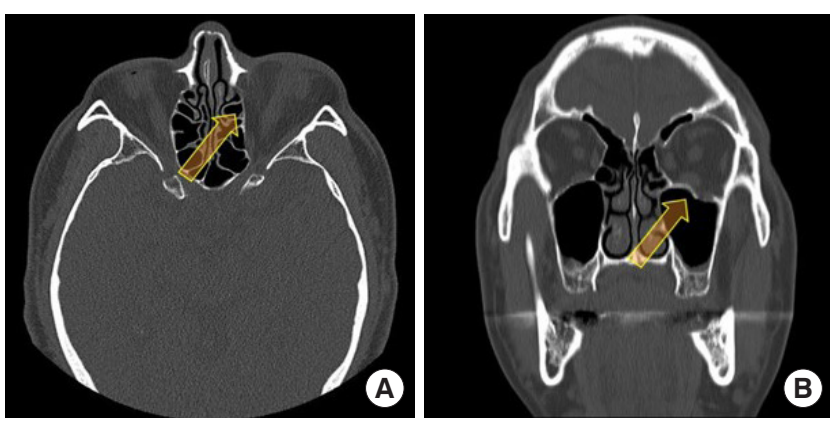

Fig. 8. Postoperative computed tomography images of case 2. Followup computed tomography at 5 days postoperatively showed removal of the mucocele (arrow). (A) Axial plane and (B) coronal plane. 
commonly develops in patients between the ages of 40 and 70 years, with no difference by sex [6]. The frontal and ethmoid sinuses are the most common sites of mucocele, accounting for $60 \%$ and $30 \%$ of patients, respectively. Mucocele of the maxillary or sphenoid sinus is rare [7-9]. Mucocele is characterized by a gradually growing chronic cystic lesion arising from a sinus, which can cause expansion of the sinus, bony erosion, and invasion of surrounding tissues, such as the orbit or nasopharynx. The sinuses are surrounded by respiratory mucosa, from which mucus is normally secreted. When the route of mucus secretion is blocked, a mucocele can form. The exact cause of mucocele is unknown, but congenital, infectious, or inflammatory origins are suspected. In addition, mucocele may occur as a result of previous surgery or trauma blocking the opening of the sinus or trapping sinus cells [3].

In particular, mucocele in the ethmoid sinus is associated with sinus surgery, and has recently been reported to be associated with endoscopic sinus resection. In such cases, mucocele can occur as a late complication after endoscopic ethmoidectomy [10]. Few reports have addressed the direct association between mucocele and endoscopic sinus surgery in the ethmoid sinus or orbit because the symptoms in such cases develop slowly. Regarding the cases described in this report, the authors believe that the cause of mucocele enlargement without a specific history other than sinus surgery was adhesion and tissue ingrowth at the opening of the mucosa, as observed using endoscopy. As it continues to produce mucus, a mucocele grows and can come into contact with the surrounding orbital bones and sinuses. Pressure caused by continued dilatation can lead to bone remodeling and erosion, and if left untreated, it can impact the orbit and nasopharyngeal and intracranial cavities. In particular, even under small amounts of pressure, a fronto-ethmoid sinus mucocele can easily expand into the orbit through the medial orbital wall. Although mucoceles generally grow slowly, they can expand rapidly if a cyst is secondarily infected. An infected mucocele can rupture like an abscess, leading to more serious complications such as meningitis, osteomyelitis, and periorbital cellulitis. Therefore, the early detection of mucocele is important in order to prevent these serious complications.

In contrast, mucocele development after an orbital fracture is believed to be caused by the growth of respiratory epithelial cells transplanted from the sinus to the orbit during surgery or via fracture [3]. Reconstruction of an orbital wall fracture provides an opportunity for ingrowth of the respiratory epithelium. Communication between the orbit and sinuses through the fracture site allows the implant to contact respiratory cells in the sinus mucosa, resulting in the formation of columnar epithelium surrounding the cyst. Orbital floor fractures are the most common source of ectopic epithelium. Medpor implants have been widely used for tissue reconstruction due to their biocompatibility, flexibility, and histological stability. Cysts most likely form in response to a chronic inflammatory response, in addition to the effect of ectopic epithelium transplantation. An implant itself-regardless of type-can act as a trigger for cyst formation [4].

There have been a few reports of orbital mucocele after orbital fracture reduction. The signs and symptoms vary depending on the size and degree of bone erosion. The most common symptoms are exophthalmos and eyeball displacement [7-9,11]. In addition, symptoms such as diplopia, palpation of mass, pain, nasal congestion, and purulent nasal secretion may occur. If it infiltrates into the orbit, the mucocele may compress the optic nerve, causing blindness or visual field defect. In case 1, the patient had undergone an ophthalmologic examination 2 years prior to presentation. At the initial examination, no further evaluation was performed, so the mucocele was not detected. Therefore, to diagnose mucocele, accurate history-taking and appropriate suspicion of mucocele are important.

Imaging examinations such as MRI or CT should be performed if an orbital mucocele is suspected. MRI can provide a good view of the orbital soft tissue, but CT is needed to identify bony and sinus structures, especially the osteomeatal unit. Therefore, it is recommended that both tests be performed, although if only one test is possible, CT is a better option for evaluating mucocele $[9,12,13]$.

Some reports have suggested that it is not necessary to remove all tissue or to perform marsupialization to allow sufficient mucus drainage [7-9]. Complete resection of the cyst remains the best treatment option. After the cyst has been exposed, surgeons should first aspirate the fluid inside to prevent mucocele rupture and to facilitate easy removal. To prevent recurrence and secondary infection, surgeons should completely remove both the implant and the underlying dense tissue [4]. As in this case of orbital mucocele, it is necessary to use an approach that provides access to areas that are difficult to treat with endoscopy alone. Several such approaches exist, including the percutaneous approach, the transconjunctival approach, and the transcranial approach $[7,9,12,13]$. In the first presented case, the authors removed the mucocele by accessing the frontal and ethmoid sinuses through a Lynch incision, which is used for nasoorbito-ethmoidal fractures. At the same time, endoscopic surgery was performed for drainage of the remnant mucocele. In the second case, a subciliary incision was made. Although such a percutaneous incision causes a scar, it is useful for complete removal of the mucocele and essential for correcting the bony structure. 
Surgeons should always suspect orbital mucocele if a patient complains of a mass effect around the orbit. Early diagnosis and surgical intervention are key for the prevention of potentially fatal complications and recurrence.

\section{NOTES}

\section{Conflict of interest}

No potential conflict of interest relevant to this article was reported.

\section{Ethical approval}

The study was approved by the Institutional Review Board of St. Vincent's Hospital (IRB No. VC19RESI0201) and performed in accordance with the principles of the Declaration of Helsinki. Written informed consents were obtained.

\section{Patient consent}

The patients provided written informed consent for the publication and the use of their images.

\section{ORCID}

Se Young Oh https://orcid.org/0000-0001-9226-8386

Ji Seon Choi https://orcid.org/0000-0002-3531-0530

Jin Soo Lim https://orcid.org/0000-0001-8406-7568

Min Cheol Kim https://orcid.org/0000-0002-2227-4374

\section{REFERENCES}

1. Koudstaal MJ, van der Wal KG, Bijvoet HW, Vincent AJ, Poublon RM. Post-trauma mucocele formation in the frontal sinus; a rationale of follow-up. Int J Oral Maxillofac Surg 2004; 33:751-4

2. Kamoshima Y, Terasaka S, Nakamaru Y, Takagi D, Fukuda S, Houkin K. Giant frontal mucocele occurring 32 years after fron- tal bone fracture: a case report. Case Rep Neurol 2012;4:34-7.

3. Park J, Kim J, Choi J, Kim H. Mucocele after orbital fracture repair masquerading as optic neuritis. J Craniofac Surg 2016;27: 1041-3.

4. Mahmoud SM, Liao HT. Endoscopic-assisted removal of a rare mucocele complicating from alloplastic medial orbital fracture reconstruction. J Craniofac Surg 2017;28:967-9.

5. Su Y, Sun J, Fan X. Epithelial cysts associated with alloplastic implants after repair of orbital fractures: a systematic review and four new cases. Br J Oral Maxillofac Surg 2016;54:658-63.

6. Nkenke E, Amann K, Maier T, Benz M, Kramer M, Haeusler G, et al. Untreated 'blow-in' fracture of the orbital floor causing a mucocele: report of an unusual late complication. J Craniomaxillofac Surg 2005;33:255-9.

7. Shah A, Meyer DR, Parnes S. Management of frontoethmoidal mucoceles with orbital extension: is primary orbital reconstruction necessary? Ophthalmic Plast Reconstr Surg 2007;23: 267-71.

8. Benkhatar H, Khettab I, Sultanik P, Laccourreye O, Bonfils P. Mucocele development after endoscopic sinus surgery for nasal polyposis: a long-term analysis. Ear Nose Throat J 2018;97: 284-94.

9. Herndon M, McMains KC, Kountakis SE. Presentation and management of extensive fronto-orbital-ethmoid mucoceles. Am J Otolaryngol 2007;28:145-7.

10. Busaba NY, Salman SD. Ethmoid mucocele as a late complication of endoscopic ethmoidectomy. Otolaryngol Head Neck Surg 2003;128:517-22.

11. Lund VJ, Rolfe ME. Ophthalmic considerations in fronto-ethmoidal mucocoeles. J Laryngol Otol 1989;103:667-9.

12. Weitzel EK, Hollier LH, Calzada G, Manolidis S. Single stage management of complex fronto-orbital mucoceles. J Craniofac Surg 2002;13:739-45.

13. Malloy KA. Fronto-ethmoid sinus mucocele: a case report. Optometry 2006;77:450-8. 